\title{
Urinary incontinence: Clinical observation on 30 patients undergoing treatment with F.R.E.M.S (Frequency Rhythmic Electrical Modulation System)
}

\author{
Massimo Massari ${ }^{1}$, Patrizia Desideri ${ }^{2}$, Paolo Menchinelli ${ }^{3}$, Lucia Cerrito ${ }^{4}$, Luciano De Giovanni ${ }^{3}$ \\ ${ }^{1}$ General Surgery Unit 1, Complesso Integrato Columbus, Catholic University of the Sacred Heart, Rome, Italy; \\ 2 Specialist Genesy Srl; \\ ${ }^{3}$ Urologic Surgery Unit, Complesso Integrato Columbus, Catholic University of the Sacred Heart, Rome, Italy; \\ ${ }^{4}$ Department of Internal Medicine, Policlinico A. Gemelli, Catholic University of the Sacred Heart, Rome, Italy.
}

\begin{abstract}
Summary Aim of the study: Urge incontinence is considered to be a dysfunctional pathology of social interest due to the psychological and relational implications of such disability, the elevated number of affected patients and the consequent treatment costs. We propose an innovative non-pharmacological and non-invasive care methodology: Frequency rhythmic electrical modulation system (F.R.E.M.S.) therapy (FT), based on the administration of electric fields of monophasic pulsed, negative, asymmetric current, generated by a neurostimulator with the characteristics of low variable frequency, high voltage and very low impulse duration.

Material and Methods: 30 patients were studied with urodynamic evaluation and radiological diagnostic techniques, and underwent 2 cycles of 15 days therapy, with a 12 months follow-up.

Results: In 93\% of cases, we obtained a positive result, with either disappearance or improvement of symptoms. Conclusion: Although the Authors believe that clinical results deserve further neurohistological and immunohistochemical studies, in order to define the anathomophysiological and biochemical changes induced by FT, they propose it as a possible alternative to traditional pharmacological therapy and electrical stimulation.
\end{abstract}

KEY WORDS: Urge incontinence; F.R.E.M.S.; Lorenz therapy; Neuromodulation; Electrostimulation.

Submitted 17 March 2015; Accepted 30 April 2015

\section{INTRODUCTION}

The term "urge incontinence" includes a symptom complex characterized by pollakiuria, urgency and urge incontinence.

In 1971 Bates gave the first definition as "bladder instability" or "detrusor instability", which became in 1988, at International Continence Society, "detrusor hyperactivity", divided in "detrusor instability" and "detrusor hyperreflexia", depending on whether or not the neurological pathology is clinically manifested.

Successively, Paul Abrams and Alan Wein created the term "overactive bladder" (OB) exclusively on a clinical- symptomatological concept. Currently, it seems reasonable to define urinary urge incontinence as the involuntary urine loss accompanied or immediately preceded by urgency; inversely, urinary stress incontinence can be described as involuntary urine loss during exertion or exercise, sneezing or coughing. Mixed incontinence is characterized by losses associated to urinary urgency and effort.

Symptomatology in women is much more frequent (43.2\% of patients affected by urinary incontinence) while in men it can be considered an occasional event with an average frequency of one episode per month (21.9\% of patients with urinary incontinence).

The overall prevalence in a population by the fourth decade of life onwards is estimated between 12 and 22\%, with about 3 million people with OB in Italy.

Despite being a dysfunctional disease, the set of symptoms and the repercussions on everyday relational life of affected patients, make it a disease of social interest due to its entity, incidence and costs (1).

Normal micturition reflex depends on a proper balance between excitatory and inhibitory descending pathways and on an afferent control coming from bladder, urethra and pelvic floor (2).

Functions related to detrusor contraction, release of bladder sphincter, contraction and relaxation of the pelvic floor muscles, are determined by the interaction of Autonomic Nervous System (ANS) and the Central Nervous System (CNS) through the spino-ponto-spinal neuronal circuit, where acetylcholine of the parasympathetic postganglionic muscarinic receptors plays an essential role in transmitting the electrical signal coming from detrusor relaxation $(3,4,5)$.

The muscle hyperexcitability, caused by the partial denervation of the detrusor, the increase of peripheral afferent nerve fibers or their reduced inhibition at different levels of micturition neural circuitry, cause the vesicourethral-pelvic functional imbalance, at the basis of $\mathrm{OB}$ symptoms.

F.R.E.M.S.Therapy (FT) is based on the delivery of electric fields at fixed and variable frequency, administered with 
a non-invasive transcutaneous technique. The electric fields of FT are generated by a monophasic pulsed negative asymmetrical current (Lorenz Therapy ${ }^{\circledR}$ ), created in the laboratory, administered in patterns of electrical signals, capable of producing depolarizing action potentials on semipermeable membranes of neuronal and muscular cells.

In particular, in vitro and in vivo studies (by Monticelli and Barrella) $(6,7)$ showed that the action of an electric field with variable frequency, is capable of creating a depolarizing action potential, in highly differentiated nerve cells and muscle cells, based on the recruitment of the highest density of voltage-gated ion channels ( $\mathrm{Ca}, \mathrm{K}$, $\mathrm{Na}, \mathrm{Mg}$ ) and neurotransmitter-dependent channels, present in semipermeable cellular membranes.

The peculiar characteristic of continuous modulation of the pulse frequency allows the sequence of pulses patterns, used by FT, to activate and modulate peripheral muscle reinnervation phenomena and adjust the electrical signals generated or transmitted by neuronal cells.

\section{Materials AND METHOdS}

In the period from January 2013 to December 2013, we selected thirty patients, 11 males and 19 females, aged between 24 and 76 years old, who came to our attention at the laboratory of Urodynamics of Integrated Complex Columbus (Urological Surgery), excluding the peacemaker bearers, patients with episodes of epilepsy, patients with a neoplasia that has been diagnosed, suspected or in course of chemotherapy. In 15 cases referred symptoms consisted only in urge incontinence, while in the remaining 15 cases there was a mix of stress and urge incontinence.

In the 15 cases of simple urge incontinence, symptoms included recurrent cystitis from at least 1 year (3 cases), referred ineffectiveness of therapy with tolterodine and oxybutynin (6 cases) at standard dosages.

In 2 cases, the symptoms developed after prostatectomy for a prostatic adenomyoma; in 3 cases it was secondary to surgical correction of cystocele and transvaginal hysterectomy.

In the 15 cases with a mix of urge and stress incontinence, symptoms were also associated with recurrent cystitis (6 cases) and suprapubic pain even at average bladder distension ( 3 cases); in 2 cases symptomatology occurred after prostatectomy for benign prostatic pathology (3 cases), in one case it had a postpartum onset with inability to stop the micturition; ineffectiveness of pharmacological therapy in 2 cases (tolterodine, oxybutynin, administered at standard doses).

In 21 cases, not associated to recurrent cystitis, X-ray cystography and pre - FT urodynamic examination (slow filling cystomanometry with control of abdominal pressure) were performed.

Cystography showed normal findings in 2 cases (10\%), in 4 cases (20\%) bladder floor was moderately under the level of the pubic symphysis, bladder prolapse in 5 cases (23\%), cystocele in 3 cases (14\%), smoothing of vesicourethral angle in 7 cases (33\%).

The cystomanometric evaluation, before FT, documented in 11 cases (52\%) the reduction in bladder capacity and compliance associated with sphincter hypotonia (6 of 11) and urinary escape (4 of 11); in 6 cases (29\%) the detrusor instability with early urinary escape; in 4 cases (19\%), the detrusor instability associated with sphincter hypotonia (1 of 4) and increase of proprioceptive sensitivity (3 of 4 ).

The electric fields of FT, generated by the neurostimulator ETS 501 (Lorenz Biotech, Medolla, Italy) were administered daily, for 15 sessions lasting 30 minutes each, by affixing the skin of pre-gelled electrodes with two methods in each session:

a) in bilateral paravertebral lumbosacral region for 10 minutes with a fixed frequency of $110 \mathrm{~Hz}$ and automatic increase of 33\% every 3 minutes, pulse duration $20 \mu \mathrm{Sec}$ and variable voltage 70-250 Volts managed by the patient with sub-threshold remote control (+/- 1 Volt); for further 10 minutes with a frequency of $420 \mathrm{~Hz}$, pulse duration $10 \mu \mathrm{Sec}$ and variable voltage 70-250 Volts managed by the patient;

b) in suprapubic region, anode-cathode distance 4-6 $\mathrm{cm}$, for a period of 10 minutes with variable frequency 1-100 Hz and variable duration 10-40 $\mu \mathrm{Sec}$ (parameters managed by a software), and variable voltage up to 250 Volts managed by the patient.

\section{Results}

In none of treated patients we recorded side effects.All patients underwent a first cycle of FT. Three patients, who did not get any benefit after the first cycle of FT, refused to submit to a second cycle.

The remaining 27 patients found partial benefit from the first cycle and required to undergo a second round of 15 daily sessions; the cycle of therapy was administered after an interval of two weeks.

At the end of this second cycle it was asked to the patients to express subjective satisfaction with the results, on a scale of four levels: very satisfied, satisfied, moderately satisfied, unsatisfied.

We recorded in 93\% of cases, 25 patients, a satisfaction for the results obtained (19 satisfied, 6 very satisfied); in particular: in 14 cases (56\%) we obtained a disappearance of urge incontinence and of recurrent cystitis episodes ( 7 cases), in 7 cases (28\%) clear improvement of urge incontinence and disappearance of burning micturition and urinary leakage; in 4 cases (16\%) improvement of urge incontinence but persistent, even if modest, suprapubic pain (2 cases) and recurrence of cystitis (2 cases). Two patients (7\%), in which urge incontinence and stress incontinence were both present, affirmed they were moderately satisfied not having achieved greater improvement, compared with that obtained after the first cycle. All 27 patients were monthly supervised in the clinical follow-up, for 12 months. After 6-8 months from the end of the second cycle of FT we recorded recurrence of urge incontinence in 3 cases out of 14 and worsening in 1 out of 4 cases where it was previously improved; recurrent cystitis with a single episode during follow up in 2 cases out of 9.

The four patients with relapse or worsening of urge incontinence, asked to undergo a further cycle of FT, obtaining clinical benefit again. 


\section{Discussion AND CONCLUSIONS}

The data derived from clinical observation and follow-up suggest the following brief reflections and conclusions.

In patients with a severe condition of functional urological disability or non-responding to traditionally used pharmacological therapies $(8,9)$, we used a simple, noninvasive method, that is repeatable, without proven side effects, which does not require complementary pharmacological therapies and that allowed us to get results with very low rates of application compared to the traditionally used electrostimulation therapies.

We achieved a high level of satisfaction with the results obtained and a low rate of relapse after a quite long healthy period, with clear improvement of psychological and relational conditions in the daily lives of patients.

The pathophysiological causes underlying the onset of overactive bladder are related: to the partial denervation or altered generation and conduction of electrical signals from the detrusor neural network, that records the distension of the muscle; to the excess of electrical signals that cover the ascending neuronal pathways of the sympathetic chain and lumbar dorsal root ganglia; the inability of the CNS in the pontine area, deputed to function regulation of micturition, to correctly perceive the ascending electrical signals, to adjust the inhibition and the consequent loss of control on micturition, through the use of the descending paragangliar pathways of the sacral plexus.

We hypothesize that FT is able, due to the frequency modulation and the peculiar morphological characteristics of the pulse, to stimulate the phenomena of reinnervation in neuronal detrusor plexus and possibly modulate the production and activation of synaptic acetylcholine, through the depolarizing activity on nerve cells, using neurotransmitter-dependent and voltage-gated ion channels, present in the semi-permeable membranes of neuronal cells.

We also assumed that FT can play a key role in the partial or complete regression of recurrent cystitis (as documented in the sample of patients that were object of this study): in fact, it has been shown to be able to modulate the synthesis and release of inflammatory cytokines (although only in experimental data).
We, therefore, planned a neurohistologic and immunohistochemical evaluation protocol in order to demonstrate the anathomo-pathological changes that FT might be able to stimulate and modulate.

\section{Bibliography}

1. CreaseyGH, Dahlberg JE. Economic consequences of an implanted neuroprosthesis for bladder and bowel management. Arch Phys Med Rehabil. 2001; 82:1520-5.

2. Buback D. The use of neuromodulation for treatment of urinary incontinence. AORNJ 2001; 73:176-8,181-7,189-90.

3. Chartier-Kastler EJ, Ruud Bosch JL, Perrigot M, et Al. Long-term results of sacral nerve stimulation (S3) for the treatment of neurogenic refractoryurge incontinence related to detrusor hyperreflexia. J Urol 2000; 164: 1476-80.

4. Janknegt RA, Hassouna MM, Siegel SW, et al. Long-term effectiveness of sacral nerve stimulation for refractory urge incontinence. Eur Urol. 2001; 39:101-6.

5. Walsh IK, Thompson T, Loughridge WG, et al. Non-invasive antidromic neurostimulation: a simple effective method for improving bladder storage. Neurourol Urodyn. 2001; 20:73-84.

6. Barrella M. Principi biofisici, meccanismo d'azione e rilievi elettrofisiologici delle correnti impiegate nella neuromodulazione. Esperienze su un metodo innovativo di stimolazione transcutanea a modulazione di frequenza e durata di impulsi. Applicazioni sul microcircolo. Atti Congr Int "Il diabete e le malattie neurologiche invalidanti” Orvieto, maggio 2004.

7. Monticelli G. Neuroni come oscillatori. Molte cellule nervose esibiscono attività elettrica periodica. Quale è la genesi di tale attività ritmica? E' possibile indurre particolari segnali periodici mediante interventi esterni? Ricerca scientifica ed educazione permanente", Università dgli Studi di Milano 1979; 6:12-17.

8. Bosch RJL e Groen J. Sacral nerve neuromodulation in the treatment of refractorymotor urge incontinence. Curr Opin Urol. 2001; 11:399-403

9. Scheepens WA, Weil Eh, Van Koeveringe GA, et al. Buttock placement of the implantable pulse generator: a new implantation technique for sacral neuromodulation-a multicenter study. Eur Urol. 2001; 40:434-8.

\author{
Correspondence \\ Massari Massimo, MD \\ massimo.massari@virgilio.it \\ Menchinelli Paolo, MD \\ p.menchinelli@libero.it \\ De Giovanni Luciano, MD (Corresponding Author) \\ lucdegiovanni@libero.it \\ Via G. Moscati, 31-33, 00168 - Roma, Italy \\ Desideri Patrizia, MD \\ patrizia.desideri@virgilio.it \\ Via Achille Papa, 7/1, 00195 - Roma, Italy \\ Lucia Cerrito, MD \\ lucia.cerrito@hotmail.it \\ Largo A. Gemelli, 8, 00168 - Roma, Italy
}

